\section{(6) OPEN ACCESS}

\title{
Agrin mediates chondrocyte homeostasis and requires both LRP4 and $\alpha$-dystroglycan to enhance cartilage formation in vitro and in vivo
}

\author{
Suzanne Eldridge, ${ }^{1}$ Giovanna Nalesso, ${ }^{1}$ Habib Ismail, ${ }^{1}$ Karin Vicente-Greco, ${ }^{2}$ \\ Panos Kabouridis, ${ }^{3}$ Manoj Ramachandran, ${ }^{4}$ Andreas Niemeier, ${ }^{5}$ Joachim Herz, ${ }^{6}$ \\ Costantino Pitzalis, ${ }^{1}$ Mauro Perretti, ${ }^{7}$ Francesco Dell'Accio ${ }^{1}$
}

\begin{abstract}
Handling editor Tore K Kvien
- Additional material is published online only. To view please visit the journal online (http://dx.doi.org/10.1136/ annrheumdis-2015-207316).
\end{abstract}

For numbered affiliations see end of article.

\section{Correspondence to}

Suzanne Eldridge, Centre for Experimental Medicine and Rheumatology, William Harvey Research Institute, Barts and the London School of Medicine and Dentistry, Queen Mary University of London, London, UK; s.e.eldridge@qmul.ac.uk

MP and FD'A are co-senior authors.

Received 16 January 2015 Revised 14 May 2015 Accepted 14 July 2015 Published Online First 19 August 2015

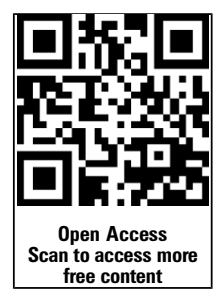

CrossMark

To cite: Eldridge $S$,

Nalesso $\mathrm{G}$, Ismail $\mathrm{H}$, et al.

Ann Rheum Dis

2016;75:1228-1235.

\section{ABSTRACT}

Objectives Osteoarthritis (OA) is a leading cause of disability for which there is no cure. The identification of molecules supporting cartilage homeostasis and regeneration is therefore a major pursuit in musculoskeletal medicine. Agrin is a heparan sulfate proteoglycan which, through binding to low-density lipoprotein receptor-related protein 4 (LRP4), is required for neuromuscular synapse formation. In other tissues, it connects the cytoskeleton to the basement membrane through binding to $\alpha$-dystroglycan. Prompted by an unexpected expression pattern, we investigated the role and receptor usage of agrin in cartilage.

Methods Agrin expression pattern was investigated in human osteoarthritic cartilage and following

destabilisation of the medial meniscus in mice.

Extracellular matrix (ECM) formation and chondrocyte differentiation was studied in gain and loss of function experiments in vitro in three-dimensional cultures and gain of function in vivo, using an ectopic cartilage formation assay in nude mice. Receptor usage was investigated by disrupting LRP4 and $\alpha$-dystroglycan by siRNA and blocking antibodies respectively.

Results Agrin was detected in normal cartilage but was progressively lost in OA. In vitro, agrin knockdown resulted in reduced glycosaminoglycan content, downregulation of the cartilage transcription factor SOX9 and other cartilage-specific ECM molecules. Conversely, exogenous agrin supported cartilage differentiation in vitro and ectopic cartilage formation in vivo. In the context of cartilage differentiation, agrin used an unusual receptor repertoire requiring both LRP4 and $\alpha$ dystroglycan.

Conclusions We have discovered that agrin strongly promotes chondrocyte differentiation and cartilage formation in vivo. Our results identify agrin as a novel potent anabolic growth factor with strong therapeutic potential in cartilage regeneration.

\section{INTRODUCTION}

Agrin (AGRN) is a heparan sulfate proteoglycan known for its requirement in neuromuscular synapse formation. ${ }^{1}$ Recent microarray data from our laboratory identified agrin as a gene expressed in cartilage and modulated upon injury, ${ }^{2}$ raising the possibility of a novel function for agrin in an aneural tissue such as cartilage, containing only one cell type: the articular chondrocyte.
At the neuromuscular junction, neuronal agrin (splice variant: $y 4, z 8$ ) binds low-density lipoprotein receptor-related protein 4 (LRP4) in a complex with the muscle-specific kinase (MuSK) and amyloid precursor protein family members APP and APLP2 resulting in MuSK activation. This then induces the aggregation of acetylcholine receptors (AChRs), ${ }^{3-5}$ ensuring neuromuscular synapse formation and maintenance. In other tissues, such as the kidneys, lung and muscle, agrin (splice variant: y0, z0) plays a role in mechanotransduction by linking the cell cytoskeleton to other basement membrane components including $\alpha$-dystroglycan (DAG1) and laminin- $\gamma 1$ (LAMC1) ${ }^{6} 7$ through either direct binding or indirectly through integrins. ${ }^{8}$ More recent studies with transgenic mice have demonstrated a non-redundant involvement of agrin in postnatal skeletal development and endochondral bone formation. ${ }^{9}$

The articular cartilage covers the ends of the long bones at the joint and ensures frictionless motion. Unlike the epiphyseal cartilage of the developing skeletal elements, which is destined to become calcified, invaded by vessels and ultimately replaced by bone through endochondral bone formation, the articular cartilage is permanent throughout life, is avascular and aneural and is resistant to calcification, vascular invasion and endochondral bone formation. Cartilage is mainly composed of specialised extracellular matrix (ECM) rich in collagen type II (COL2A1) and highly sulfated proteoglycans, prevalently aggrecan (ACAN), and chondrocytes which are sparsely present throughout the abundant ECM. ${ }^{10}$ Chondrocyte differentiation and expression of COL2A1 and ACAN is driven by the cartilagespecific transcription factor SOX9. ${ }^{11}$

Under resting conditions, cartilage matrix has a very low turnover (the half-life of COL2A1 is estimated to be $\sim 117$ years ${ }^{12}$ ); however, following damage, the tissue deploys a homeostatic response, supported by SOX9 upregulation and activity, driving the replacement of the damaged ECM and restoring cartilage integrity. ${ }^{2} 13$ When this response is insufficient or stunted, ECM breakdown takes place due to the release of proteolytic enzymes including matrix metalloproteinases (MMPs) and aggrecanases. ${ }^{14}$ Cartilage destruction and the resulting joint failure are the hallmark of osteoarthritis (OA), the most common cause of 
chronic disability worldwide after cardiovascular disease. ${ }^{15} 16$ During OA, chondrocytes acquire a phenotype similar to the hypertrophic chondrocytes of the developing growth plate, characterised by expression of collagen type X (COL10A1) and MMP-13. ${ }^{17} 18$ The acquisition of this phenotype and the consequent mineralisation drive cartilage breakdown. ${ }^{19}$ Therefore, hypertrophic differentiation and calcification are important mechanisms in OA progression. In spite of the huge social and economic burden, we still have no cure for OA and strategies for cartilage regeneration represent a priority in medical research.

In this study we reveal a novel cell-autonomous reparative autocrine/paracrine circuit with great therapeutic potential that is governed by the homeostatic function of agrin in adult articular chondrocytes.

\section{MATERIALS AND METHODS}

\section{Destabilisation of the medial meniscus}

OA was induced in 8 -week-old $129 / \mathrm{S}_{\mathrm{v}}$ male mice by surgical destabilisation of the medial meniscus (DMM) or sham surgery to the contralateral limb as described previously ${ }^{20}$ and killed after 8 weeks.

\section{Cartilage harvest and chondrocyte isolation}

Adult human articular cartilage was obtained from patients undergoing joint replacement for knee OA after obtaining informed consent. All procedures were approved by the East London and The City Research Ethics Committee. Full thickness cartilage was dissected from preserved areas of the femoral condyles and the patellar groove. From each sample, one full thickness specimen was processed for histological grading. Bovine chondrocytes were isolated from the metatarsal joints of 18-month-old bovine obtained within $6 \mathrm{~h}$ of death from a local abattoir. Full thickness sections were harvested for histological analysis. Chondrocyte isolation and in vitro expansion was performed as previously described. ${ }^{21}$

The full thickness explants taken for histological scoring were fixed in $4 \%$ paraformaldehyde in phosphate-buffered saline (PBS) at $\mathrm{pH}$ 7.4, paraffin embedded, sectioned, stained and scored for features of $\mathrm{OA}$ as described previously. ${ }^{22}{ }^{23}$ All experiments were performed using freshly isolated or confluent P0 cells.

\section{Overexpression and siRNA transfection}

Subconfluent cells were transfected with a plasmid encoding agrin (COS7 and agrin plasmid were a kind gift from Dr M Ferns, UC Davis Health System, USA), green fluorescent protein (GFP), bone morphogenetic protein (BMP)-2 (kind gift from Dr Gerard Gross, Signaling and Gene Regulation, Gesellschaft für Biotechnologische Forschung (GBF), Braunschweig, Germany), LRP4 or siRNAs $(20 \mathrm{nM})$ by using JetPrime transfection reagent (PolyPlus Transfection, Illkirch, France) according to the manufacturer's instructions. The cells were used in monolayer or micromass. Observed transfection efficiency was 25\%-30\% for C28/I2 and bovine chondrocytes; and around 95\% for COS7 cells. siRNA oligo sequences can be found in online supplementary table S1. A Stealth RNAi negative control duplex of low guanine-cytosine (GC) content (Invitrogen) was used as a negative control for agrin siRNA.

\section{Micromass culture and Alcian blue staining}

Cells were resuspended at a density of $2.0 \times 10^{7}$ cells $/ \mathrm{mL}$ in complete medium, and micromass cultures were obtained by pipetting $15 \mu \mathrm{L}$ drops of cell suspension into each well of a 24 well plate. The cells were allowed to attach for $3 \mathrm{~h}$ and then $1 \mathrm{~mL}$ of complete medium was added. Micromasses were cultured for 4 days, changing the medium every $48 \mathrm{~h}$. Micromasses were harvested for RT-PCR gene expression analysis or fixed and wholemount stained with Alcian blue. ${ }^{24}$ Alcian blue extraction and quantification was performed as previously described. ${ }^{25}$ Images were acquired at room temperature with a camera (Coolpix 4500; Nikon), with the macrosetting and daylight. Image contrast was modified with Photoshop 7.0 for best graphic rendering, equally for all treatments.

\section{Indirect immunofluorescence}

Immunocytochemistry and immunohistochemistry were performed as previously described. ${ }^{25}$ For antigen retrieval on paraffin sections we used pepsin digestion. ${ }^{25}$ The primary anti-agrin rabbit polyclonal antibody (Santa Cruz Biotechnology, Dallas, Texas, USA) or the isotype-matched negative control normal rabbit IgG (Dako, Ely, Cambridgeshire, UK) was diluted 1:200 in blocking buffer. Secondary antibodies were cy3-conjugated goat anti-rabbit antibody diluted 1:300 (Jackson ImmunoResearch Laboratories, West Grove, Pennsylvania, USA) for immunocytochemistry or goat anti-rabbit antibody Alexa 555 diluted 1:300 (Life Technologies) for immunohistochemistry. Slides were mounted in Mowiol (EMD Millipore, Darmstadt, Germany), and images were acquired with a fluorescence microscope (BX61; Olympus, Southend-on-Sea, UK) using a Uplan-Fluor $40 \times$ NA 0.85 objective lens. Images were acquired by using an F-View II Soft Imaging Solutions (SIS) camera and Cell P software (Olympus). After acquisition, the contrast of the images was enhanced for best graphic rendering using Photoshop 7.0, all with the same parameters, without altering the relationship of the target to control the images.

\section{Total RNA extraction and real-time qPCR}

Total RNA extraction, cDNA synthesis and real-time PCR were performed as previously described. ${ }^{25}$ Real-time qPCR and sequencing primers can be found in online supplementary tables $\mathrm{S} 2$ and S3.

\section{In vivo ectopic cartilage formation assay}

The ectopic cartilage formation assay was performed as described previously. ${ }^{21}$ In brief, for each injection 5 million freshly isolated bovine chondrocytes were mixed with either 500000 growth-arrested (treated with $7.5 \mu \mathrm{g} / \mathrm{mL}$ of mitomycin-C (Sigma-Aldrich) diluted in complete medium for $2 \mathrm{~h}$ at $37^{\circ} \mathrm{C}$ ) COS7 cells overexpressing FL-agrin or with the same amount of mitomycin C-treated GFP transfected COS7 cells. Each sample was resuspended in $50 \mu \mathrm{L}$ of sterile PBS and injected intramuscularly in the posterior compartment of the thigh of 3 -week-old female CD1nu/nu mice. ${ }^{23}$ Twelve injections per condition (24 in total) were performed. Animals were maintained in isolator cages under an unrestricted diet. The animals were then killed after 2 weeks and the cartilage implants were retrieved. All procedures were approved by the Local Ethics committee and the UK Home Office. The implants were weighed and then paraffin embedded for histological analysis.

\section{$\boldsymbol{\alpha}$-dystroglycan blocking}

$\alpha$-dystroglycan binding was inhibited in vitro on bovine chondrocytes in monolayer using $10 \mathrm{ng} / \mathrm{mL} \alpha$-dystroglycan antibody (IIH6; Santa Cruz). Binding inhibition was confirmed using Phalloidin (Invitrogen) staining in PBS 1:1000. IgM (Santa Cruz) was used as control antibody. 


\section{Statistical analysis}

Parametric data were compared with a student's $t$ test. $p$ Values $<0.05$ were considered significant. * $p<0.05 ; * \mathrm{*} p<0.005$; $* * * \mathrm{p}<0.0005$. All the data in the graphs are expressed as mean \pm SEM.

\section{RESULTS}

Agrin $(\mathrm{y} 0, \mathrm{z} 0)$ is expressed in the articular cartilage and is downregulated in $\mathrm{OA}$

In the context of a transcriptomic analysis to identify genes regulated by mechanical injury in cartilage, ${ }^{2}$ we discovered that
mRNA encoding for agrin and its receptors LRP4, APP, APLP2, DAG1 and LAMC1 were expressed in human adult articular cartilage (figure 1A) raising the question of whether agrin signalling has a role in cartilage biology. MuSK, which is essential at the neuromuscular junction, was not detected in two of three samples.

At the neuromuscular junction, the presence of the alternatively spliced inserts y and $\mathrm{z}$ results in a 1000 -fold higher affinity of agrin to LRP4 and therefore neuromuscular synapse stability via AChR clustering. ${ }^{4}$ To investigate whether these inserts ( $\mathrm{y} 4$ : KSRK; $\quad \mathrm{z} 8$ :ELTNEIPA or 11:PETLDRSALFS or 19:
A

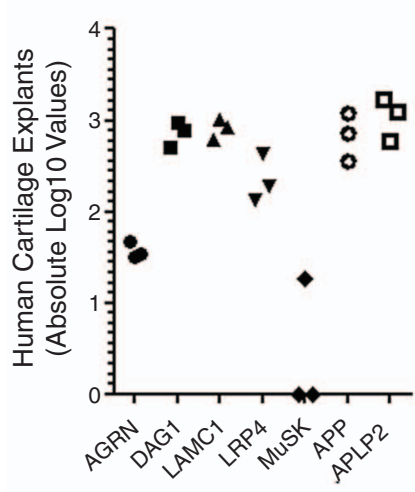
Key: GAG chains: (
Human

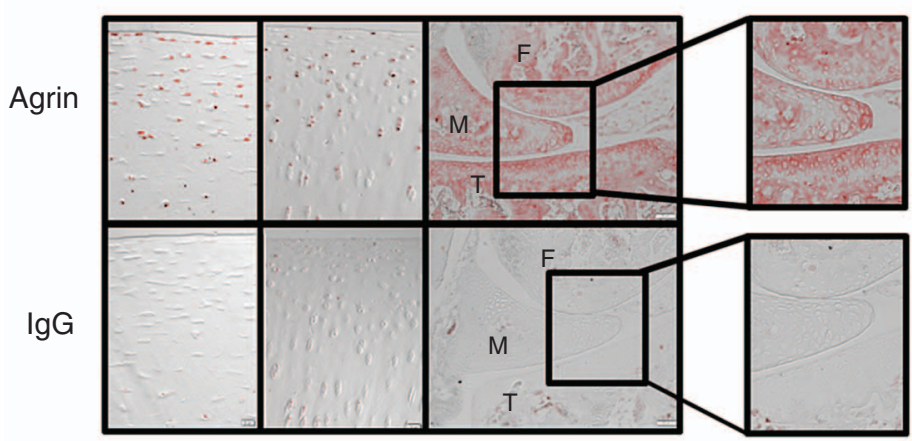

E

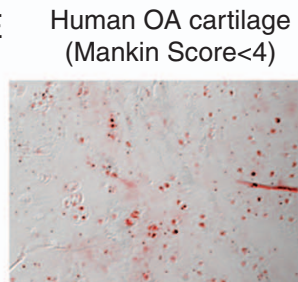

B

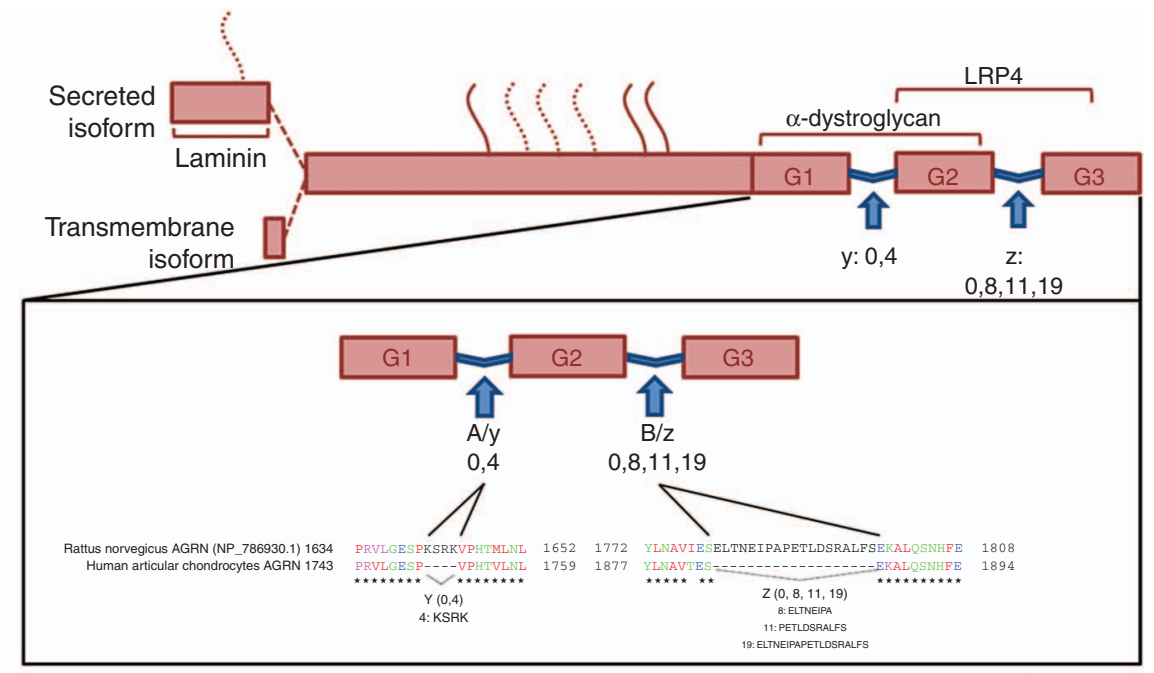

Binding sites

$\mathrm{N}$-glycosylation sites

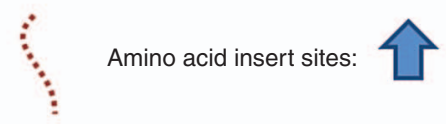

D

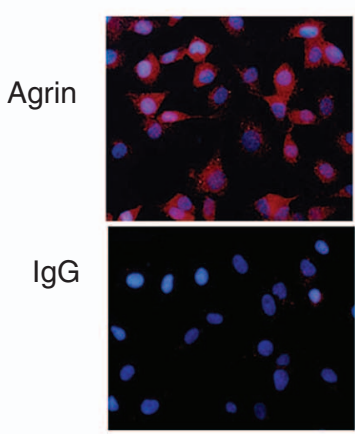

7day DMM

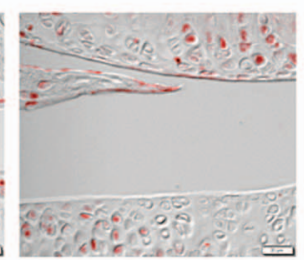

$\mathrm{C} 28 / \mathrm{l2}$

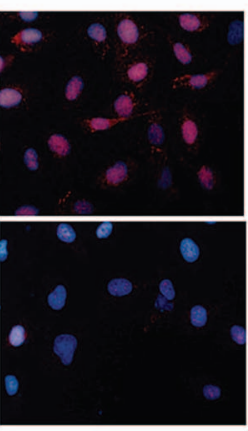

8wk DMM

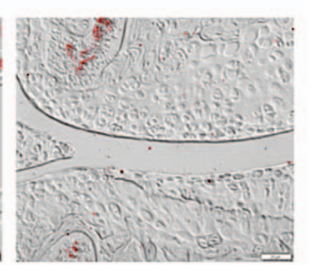

Figure 1 Agrin $(y 0, z 0)$ is expressed in the articular cartilage and reduced in OA. (A) Microarray analysis of agrin and its receptors DAG1, LAMC1, LRP4, MuSK, APP and APLP2 in freshly dissected human articular cartilage samples. (B) Schematic representation of agrin (above) and sequence analysis of agrin cDNA from human articular chondrocytes demonstrating the absence of the insert y and $z$ (y0, z0) (below). (C) Agrin protein detection in healthy human and bovine explants and in the murine joint of 129/Sv mice ( $n=3)$. F, femur; M, meniscus; T, tibia. (D) Agrin protein detected on in isolated bovine articular chondrocytes and in C28/I2. (E) Agrin protein detection in paired human cartilage samples obtained from the same joint, but either from persevered areas (Mankin score $<4$ ) or from severely affected areas (Mankin Score $>8)(n=3)$. (F) Agrin protein detection at 7 days and 8 weeks post DMM surgery in the joints of male 129/Sv mice; contralateral sham-operated joints served as control ( $n=3$ ). AGRN, agrin; DAG1, $\alpha$-dystroglycan; DMM, destabilisation of the medial meniscus; GAG, glycosaminoglycan; LAMC1, laminin- $\gamma 1$; LRP4, low-density lipoprotein receptor-related protein 4; MuSK, muscle-specific kinase; OA, osteoarthritis. 
ELTNEIPAPETLDRSALFS) were present in chondrocytes, we sequenced across the potential insert sites of agrin from human articular chondrocyte cDNA. This analysis revealed, as in other non-neural tissues, the absence of the alternatively spliced y and $\mathrm{z}$ inserts in chondrocytes (figure 1B), where agrin has a preferred, but not exclusive, affinity for dystroglycan binding compared with the $\mathrm{y} 4, \mathrm{z} 8$ isoform. ${ }^{26}$

To investigate the expression pattern of agrin in normal joints we used immunofluorescence. Agrin was abundantly detected in human, bovine and murine cartilage and menisci (figure 1C); agrin protein was retained in vitro in isolated primary bovine chondrocytes and in the human chondrocyte cell line C28/I $2^{27}$ in monolayer (figure 1D) and in three-dimensional culture (see online supplementary figure S1). In the mouse, agrin was detected within the superficial and deep layers of the articular cartilage, within the osteoid in the subchondral bone, and in the menisci. In healthy human and bovine cartilage agrin expression was distributed uniformly. However, in human OA cartilage, agrin expression was markedly reduced (almost undetectable) in the more severely affected areas (Mankin score $>8$ ) compared with the relatively preserved ones (Mankin score $<4$ ) (figure $1 \mathrm{E})$. In a validated murine model of $\mathrm{OA}$ induced by surgical $\mathrm{DMM}^{20}$ agrin expression was rapidly reduced in the articular cartilage as early as 7 days after surgery, reaching undetectable levels after 8 weeks (figure $1 \mathrm{~F}$ ).

\section{Agrin cell autonomously regulates the chondrocyte transcription factor SOX9}

The downregulation of agrin in OA cartilage prompted us to investigate whether agrin could have a function in chondrocyte differentiation and ECM formation. To investigate whether agrin has a cell-autonomous function in chondrocytes we applied a loss of function approach. Silencing agrin in C28/I2 chondrocytes using either of two independent siRNA oligonucleotide pairs (siRNA-1 and siRNA-2), we obtained a reduction of agrin mRNA by $59 \%$ and $78 \%$ respectively, allowing dose-response experiments (figure 2A). Agrin knockdown resulted in a dose-dependent decrease of absolute sulfated glycosaminoglycan (GAG) content in C28/I2 micromass cultures as evaluated by Alcian blue staining ${ }^{24}$ (figure 2B). Total DNA content was not changed, thereby suggesting that such difference did not result from a decreased cell number (figure $2 \mathrm{C}$ ).
Agrin silencing also resulted in reduced expression of the cartilage transcription factor SOX9 mRNA (figure 2D) and dosedependent downregulation of its transcriptional targets COL2A1 and ACAN mRNA (figure 2E, F).

Conversely, overexpression of full length agrin $(\mathrm{y} 0, \mathrm{z} 0)$ in bovine primary chondrocytes resulted in significantly increased sulfated GAG content compared with GFP-transfected controls, both as total amount per micromass (figure $3 \mathrm{~A}$ ) and when normalised for DNA content (figure 3B). Moreover, after 5 days culture, SOX9 mRNA was significantly upregulated and 2 days later so were its direct transcriptional targets COL2A1 and ACAN (figure 3C-E). ${ }^{28}$

These data also demonstrate that agrin function is cell autonomous as it takes place in vitro, in the absence of potential exogenous sources of agrin (eg, nerves).

\section{Exogenous agrin supports formation of stable, hyaline-like cartilage in vivo}

In vitro cartilage formation is not always predictive of cartilage formation in vivo. ${ }^{29}$ For instance, mesenchymal stem cells can form cartilage in vitro, yet fail to form stable cartilage tissue in vivo even following in vitro differentiation. ${ }^{29}$ To confirm the anabolic function of agrin in vivo, we used a highly stringent transplantation model in which bovine articular chondrocytes form stable hyaline cartilage resistant to vascular invasion and endochondral bone formation when injected ectopically, in suspension, into the muscle of immunodeficient mice. ${ }^{23} 29$ This validated model predicts clinical efficacy of cell preparations for cartilage repair in humans, ${ }^{30}$ and allows robust quantification and monitoring of gene expression. ${ }^{23}$

In order to administer exogenous agrin to the implanted chondrocytes for the entire duration of the assay we used a previously validated strategy ${ }^{4}$ based on co-implantation of growth-arrested, agrin-overexpressing COS7 cells (ratio 10:1) or with GFP-overexpressing COS7 cells as control (see online supplementary figure S3). Two weeks after implantation, the implants exposed to agrin were significantly larger than those exposed to GFP (figure 4A-C) as determined by histomorphometry (figure 4A, B) and wet weight (figure 4C) and with a larger differentiated area as determined by increased area of Safranin O metachromatic staining (figure 4A, D), but without evidence of excessive accumulation of proteoglycans (figure 4E).
Figure 2 Agrin silencing by siRNA in C28/12 resulted in reduced glycosaminoglycan content and reduced expression of chondrocyte differentiation markers. (A) Real-time PCR analysis showing agrin knockdown efficiency with agrin siRNA1 or SiRNA2 in C28/I2 chondrocytes; scrambled siRNA served as control. (B) Micromass staining with Alcian blue and spectrophotometric quantification following guanidine extraction expressed as total value (C) and normalised by DNA content. (D-F) Real-time PCR analysis of SOX9, ACAN and COL2A1 mRNA expression. Data are mean \pm SEM, $n=4 .{ }^{*} \mathrm{p}<0.05$; ${ }^{* *} \mathrm{p}<0.005 ;{ }^{* * *} \mathrm{p}<0.0005$ (unpaired t test). ACAN, aggrecan; AGRN, agrin; COL2A1, collagen type II.
A
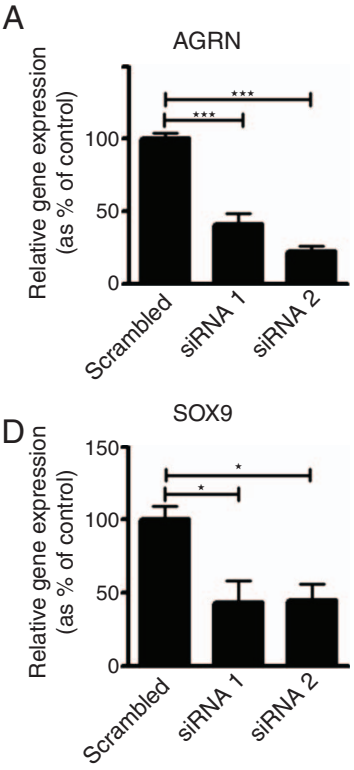
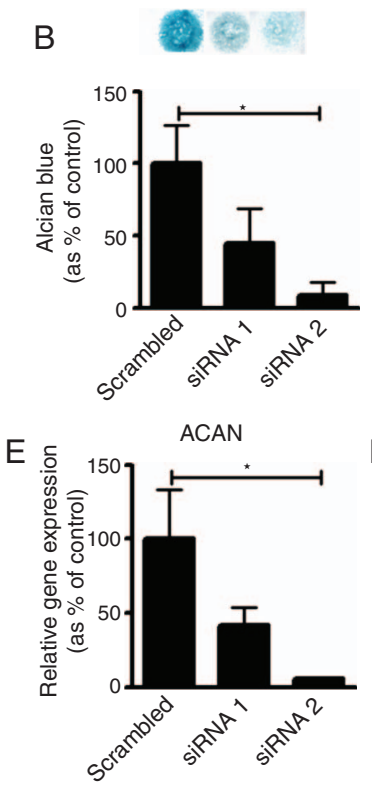

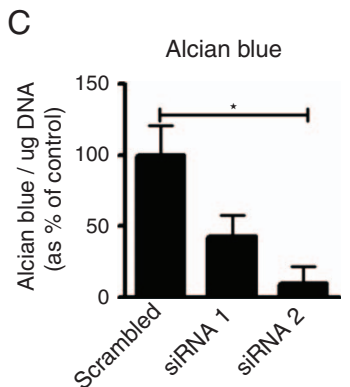

$\mathrm{F}$

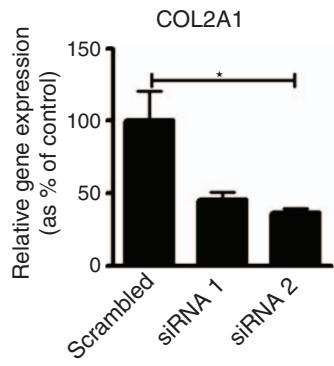


Figure 3 Agrin $(\mathrm{y} 0, \mathrm{z} 0)$ overexpression resulted in increased glycosaminoglycan production and an upregulation of chondrocyte differentiation markers. Primary bovine chondrocytes were transfected with an agrin, BMP-2 or GFP encoding expression plasmid and cultured in micromass for the time indicated. (A) Alcian blue staining and quantification following guanidine extraction expressed as total value and (B) normalised by DNA content 5 days after transfection. (C-E) Real-time PCR analysis of SOX9, ACAN and COL2A1 mRNA expression levels over 5 days (SOX9) and 7 days (COL2A1 and ACAN) after transfection. Data are mean \pm SEM, $n=4$. * $p<0.05$; ${ }^{* *} \mathrm{p}<0.005 ;{ }^{* * *} \mathrm{p}<0.0005$ (unpaired $\mathrm{t}$ test). ACAN, aggrecan; BMP-2, bone morphogenetic protein-2; COL2A1, collagen type II; GFP, green fluorescent protein
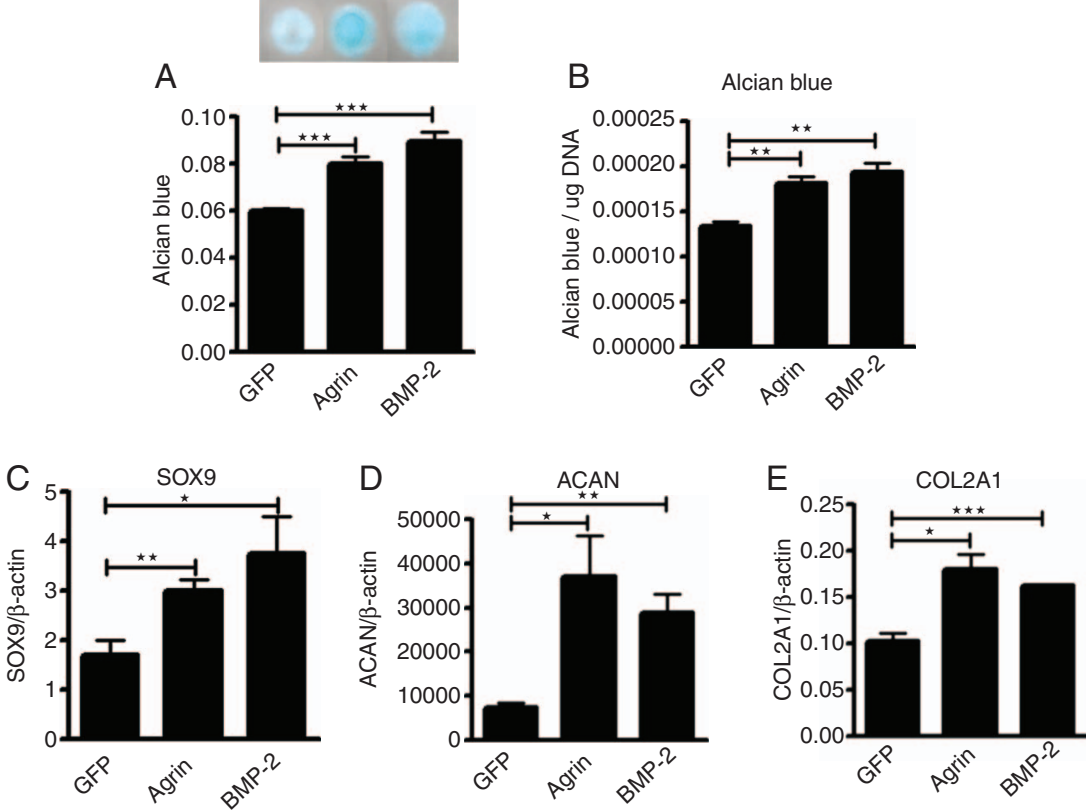

Similar data were obtained using toluidine blue staining (figure $4 \mathrm{H})$. No vascular invasion was detected with Masson's trichrome staining and no calcification was detected with alizarin red staining (figure 4I, J). Gene expression analysis performed with bovine-specific primers showed no increased expression of COL10A1 and MMP-13 in the agrin-treated group compared with control (figure $4 \mathrm{~F}, \mathrm{G})$.

\section{Agrin-induced upregulation of SOX9 is $\alpha$-dystroglycan and LRP4 dependent}

At the neuromuscular junction, neuronal agrin $(\mathrm{y} 4, \mathrm{z} 8)$ binds to LRP4 and signals through MuSK, while in muscle cells, nonneuronal agrin $(\mathrm{y} 0, \mathrm{z} 0)$ links the cytoskeleton to the basement membrane through binding $\alpha$-dystroglycan. ${ }^{31}{ }^{32}$ Therefore we investigated which of these two mechanisms was engaged for the anabolic functions of agrin in chondrocytes. Treatment with an antibody which blocks the interaction of $\alpha$-dystroglycan with agrin $^{43}$ abrogated agrin-induced upregulation of SOX9 (figure 5A). Surprisingly, also LRP4 silencing by siRNA completely inhibited agrin-induced SOX9 upregulation (figure 5B), suggesting that both LRP4 and $\alpha$-dystroglycan are required for agrin-induced chondrocyte differentiation.

The requirement of LRP4 is unexpected because the (y0, z0) variant of agrin has low affinity for LRP4 and a reduced capacity for clustering the AChR at the neuromuscular junction. ${ }^{34}$ However, overexpression of LRP4 alone also induced SOX9 expression in primary bovine chondrocytes (figure 5C), in keeping with a previous report in ATDC5 cells. ${ }^{35}$ This and the fact that deficiency of LRP4 result in a skeletal phenotype ${ }^{36}$ support a role for LRP4 in chondrocytic differentiation. Interestingly, the overexpression or silencing of agrin in chondrocytes did not regulate the mRNA expression of LRP4 (figure $5 \mathrm{D}, \mathrm{E})$.

\section{DISCUSSION}

We have discovered that the $(\mathrm{y} 0, \mathrm{z} 0)$ isoform of agrin is expressed in the adult articular cartilage and is downregulated in OA. We showed that agrin has an anabolic function in maintaining chondrocyte differentiation and enhancing ECM production in vitro and in vivo without inducing chondrocyte hypertrophy or endochondral bone formation. We have found that agrin-induced chondrocyte differentiation requires a unique receptor/coreceptor repertoire including both $\alpha$-dystroglycan and LRP4 in articular chondrocytes.

Agrin function at the neuromuscular synapse requires the binding of LRP4 and, indirectly, MuSK, ${ }^{3} 37$ whereas the function of agrin in muscle cells is mediated by its binding to $\alpha$-dystroglycan and laminin. It is thought that the differential binding to these different receptor complexes is dependent on the presence of the spliced inserts $y$ and $z$, which confer high affinity for LRP4. ${ }^{26}{ }^{38}$ In particular, structural data of the agrinLRP4-MuSK complex revealed that the z8 loop of rat neuronal agrin was responsible for the primary agrin-LRP4 interaction, preceding the formation of the tetramer in which two agrinLRP4 heterodimers interact and for MuSK-dependent signalling at the neuromuscular junction. ${ }^{39}$ However, we previously showed that the engagement of the coreceptors APP and APLP2 (also expressed by chondrocytes-figure 1A) can independently interact with the $\mathrm{z} 0$ form of agrin as well as with LRP4, ${ }^{3}$ to either stabilise the interaction of agrin with LRP4 or indirectly recruit LRP4 into a larger agrin signalling complex and thereby support its biological function as a BMP and WNT (Wingless type MMTV integration site family member) signal modulator. ${ }^{40}$ Taken together, these data suggest that an extended cell surface receptor repertoire regulates the affinity of agrin for different receptors and the interplay with other signalling pathways including BMPs and WNTs to fine-tune biological specificity in the maintenance of articular cartilage.

In the skeletal context, as hypothesised in figure 6, LRP4 appears to modulate and coordinate fundamental signalling pathways including WNTs and BMPs. While both these pathways are essential for the homeostasis and integrity of cartilage throughout life, ${ }^{41-44}$ their excessive/inappropriate activation drives cartilage breakdown. ${ }^{45}{ }^{46}$ In humans, single nucleotide polymorphisms in genes involved in the WNT and BMP pathways are associated with predisposition to develop OA. ${ }^{47} 48$ This modulation/coordination may take place through a multitude of potential mechanisms including LRP4 acting as a receptor for the WNT and BMP inhibitors sclerostin ${ }^{33}$ and Wise, ${ }^{40}$ as well as the WNT inhibitor Dickkopf (DKK)- $1,{ }^{33}$ competing 
A

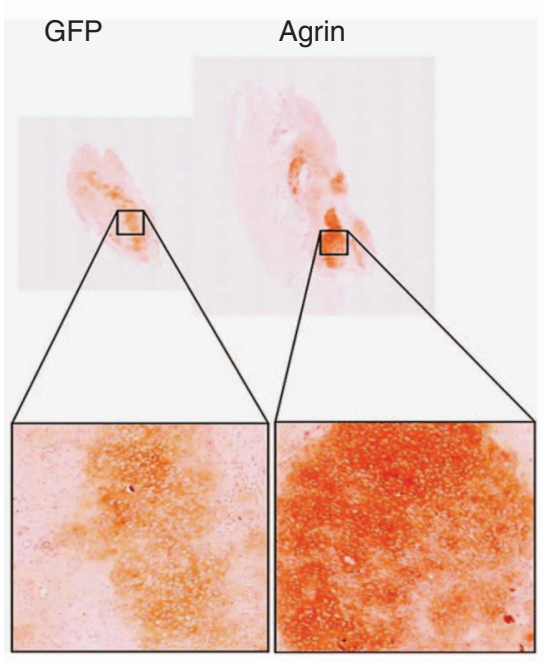

Positive control: Murine forepaw E18.5

$\mathrm{H}$

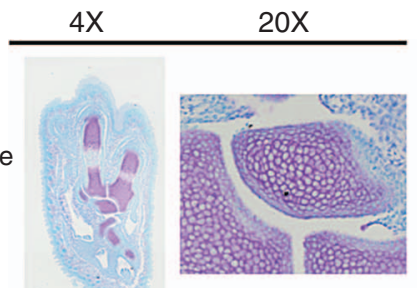

I
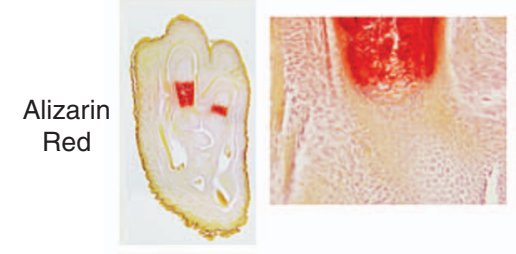

$J$

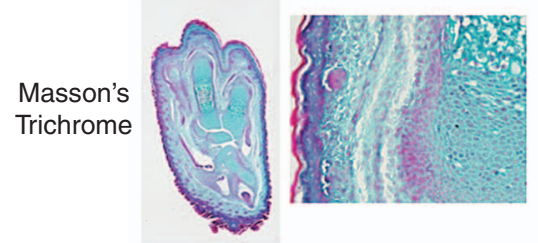

B

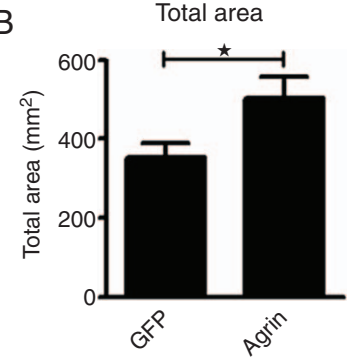

D
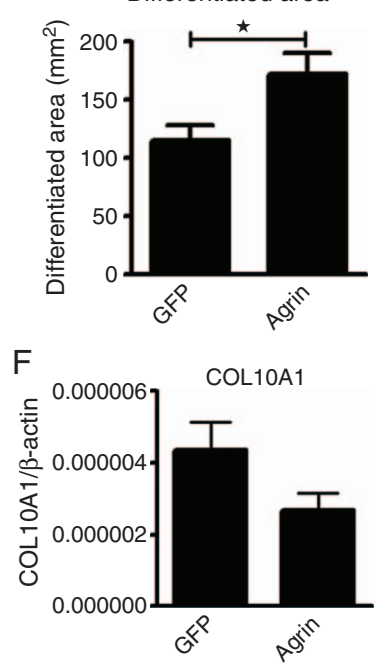

C

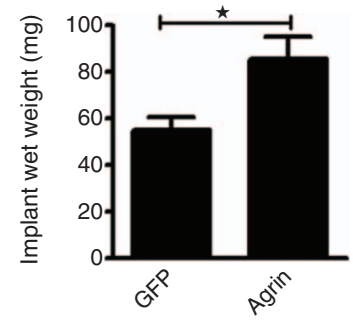

E

E Normalised differentiated area
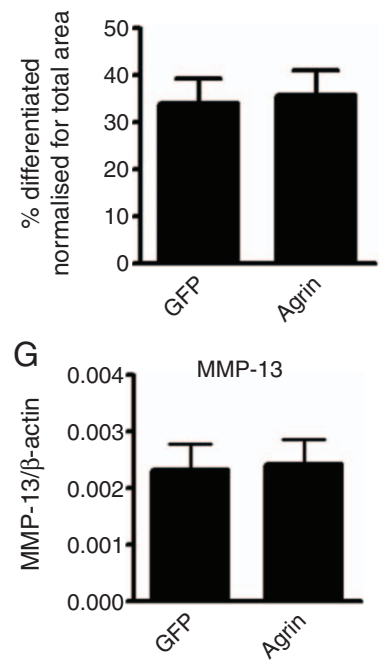

Agrin-treated

Figure 4 Exogenous agrin (y0, z0) enhanced ectopic cartilage formation in vivo. Primary bovine chondrocytes were co-implanted in suspension within the muscle of the thigh of nude mice with growth arrested COS7 cells overexpressing GFP or agrin. Cartilage implants were retrieved after 2 weeks. (A) Safranin 0 staining showing increased accumulation of metachromatic cartilage matrix in the implants supplemented with agrin. (B-E) Histomorphometric analysis of the implant wet weight, total area, differentiated area and differentiated area normalised for total area shown as a percentage. ( $F$ and $G$ ) Real-time PCR analysis of COL10A1 and MMP-13 mRNA expression levels of the implants using bovinespecific primers. (H-J) Histochemical staining was performed to confirm the presence of cartilage-specific highly sulfated glycosaminoglycans (toluidine blue) and the absence of calcification (alizarin red) and vascular invasion (Masson's trichrome); E18.5 murine forepaw was used as control. Data are mean $\pm S E M, n=12$. ${ }^{*} p<0.05 ;{ }^{* *} p<0.005 ;{ }^{* *} p<0.0005$ (unpaired t test). GFP, green fluorescent protein; MMP, matrix metalloproteinase. 

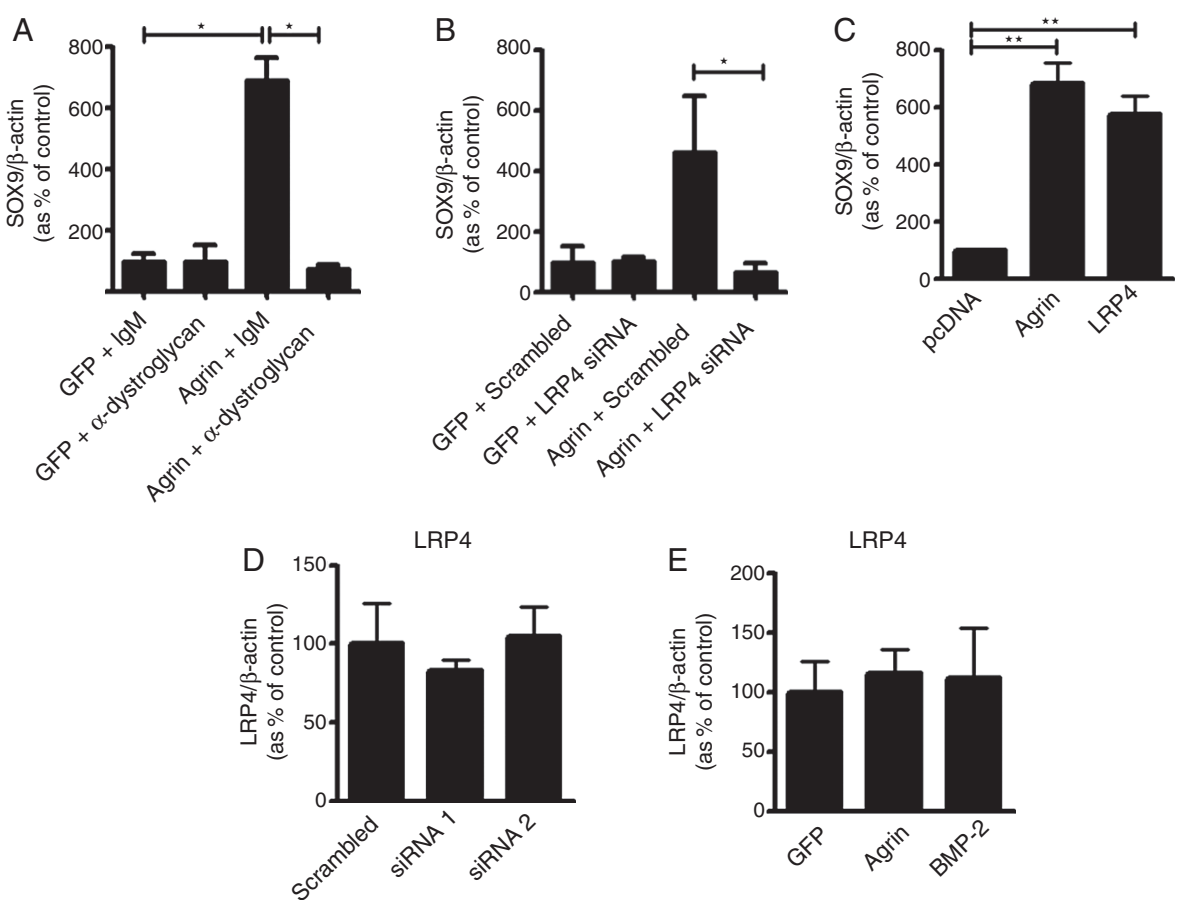

Figure 5 LRP4 and $\alpha$-dystroglycan are both required for agrin-induced upregulation of SOX9. (A) Primary bovine chondrocytes were transfected with an agrin expression plasmid or GFP as control and exposed to $\alpha$-dystroglycan blocking antibody or a non-immune isotype control antibody and assessed 5 days later for the expression of SOX9 mRNA by real-time PCR. (B) Real-time PCR analysis of SOX9 mRNA expression in chondrocytes transfected with agrin or GFP in combination with LRP4 siRNA or scrambled siRNA as control. (C) mRNA levels of SOX9 in bovine primary chondrocytes transfected with agrin or LRP4. Empty plasmid pcDNA was used as vehicle control. (D) Real-time qPCR analysis of LRP4 mRNA when agrin is silenced by siRNA and (E) when agrin is overexpressed. For validation of $\alpha$-dystroglycan blocking antibody and LRP4 siRNA efficacy see online supplementary figure S2. Data are mean $\pm \mathrm{SEM}, \mathrm{N}=4$. ${ }^{*} \mathrm{p}<0.05 ;{ }^{* *} \mathrm{p}<0.005 ;{ }^{* *} \mathrm{p}<0.0005 ;{ }^{* * * *} \mathrm{p}<0.0001$ (unpaired t test). BMP-2, bone morphogenetic protein-2; GFP, green fluorescent protein; LRP4, low-density lipoprotein receptor-related protein 4.

Figure 6 Agrin and LRP4 are central to a chondrogenic signalling network. Agrin is a paracrine signal which supports chondrocyte differentiation through a pathway requiring both LRP4 and $\alpha$-dystroglycan which upregulates SOX9 and its transcriptional targets COL2A1 and ACAN. Agrin-induced chondrocyte differentiation is not associated with chondrocyte hypertrophy. LRP4 interacts with other pathways regulating chondrocyte differentiation including WNTs and BMPs. ${ }^{35} 4046$ ACAN, aggrecan; BMPs, bone morphogenetic proteins; COL2A1, collagen type II; FZD; frizzled receptor; LRP, low-density lipoprotein receptor-related protein; MMP, matrix metalloproteinase.

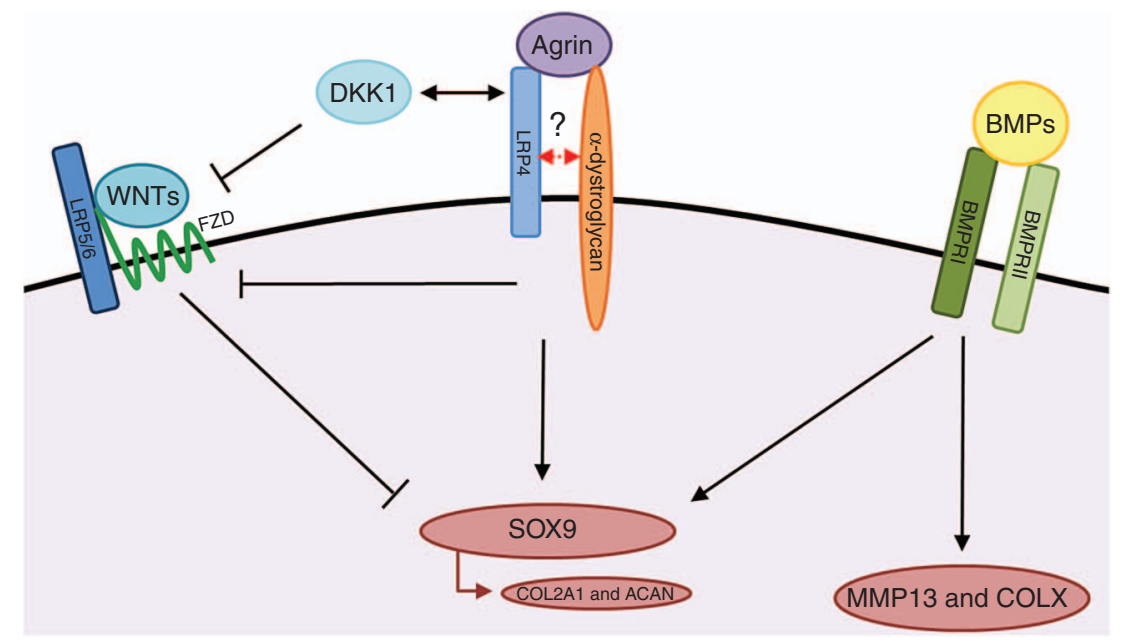

with the WNT coreceptors LRP5 and LRP6 and with the intracellular WNT machinery. The overexpression of LRP4 alone in primary articular chondrocytes was sufficient to increase the expression of SOX9 (figure 5C) in primary chondrocytes, supported chondrogenic differentiation and inhibited WNT signalling in the ATDC5 cell line; ${ }^{35}$ in vivo, mice carrying a hypomorphic LRP4 allele developed a significant skeletal phenotype. $^{33} 36$

The identification of a specific coreceptor repertoire and of a specific agonist, agrin, capable of promoting the formation of phenotypically stable hyaline-like cartilage resistant to vascular invasion and endochondral bone formation in vivo, is a unique therapeutic opportunity in cartilage-regenerative medicine and in the field of OA and opens a new exciting avenue of investigation to understand how high specificity of a biological effect is achieved by modulating otherwise pleiotropic signalling pathways.

\section{Author affiliations}

${ }^{1}$ Centre for Experimental Medicine and Rheumatology, William Harvey Research Institute, Barts and the London School of Medicine and Dentistry, Queen Mary University of London, London, UK

${ }^{2}$ Department of Surgical Research, NPIMR Y3, Harrow, UK

${ }^{3}$ The Francis Crick Institute (Mill Hill Laboratory), The Ridgeway, London, UK

${ }^{4}$ Department of Trauma and Orthopaedic Surgery, Royal London Hospital,

London, UK

${ }^{5}$ Department of Orthopaedics and IBMII: Molecular Cell Biology, University Medical Center Hamburg-Eppendorf, Hamburg, Germany 
${ }^{6}$ Department of Molecular Genetics, Center for Translational Neurodegeneration Research, University of Texas Southwestern Medical Center, Dallas, Texas, USA ${ }^{7}$ Centre for Biochemical Pharmacology, William Harvey Research Institute, London, UK

Acknowledgements We thank Dr M Ferns (UC Davis Health System, USA) for providing the Agrin plasmid and Dr G Gross (Signaling and Gene Regulation, Gesellschaft für Biotechnologische Forschung (GBF), Braunschweig, Germany) for providing the BMP-2 plasmid; Mr Y Pengas, Mr P Achan and Mr M Ramachandran for the supply of human knee samples (Barts and the London National Health Service Trust, London, England, UK). We thank Dr Mary Goldring for the C28/12 cell line.

Contributors SE, FD, KV-G, PK, GN and MP designed the experiments, analysed the data and prepared the manuscript. Experimental work was performed by $\mathrm{SE}$, with contributions from GN, HI and KV-G. FD, MR, JH, AN, PK, MP and CP contributed to experimental design and writing of the manuscript.

Funding We gratefully acknowledge funding support of this work by the MRC (MR/ L022893/1, MR/K013076/1 and G1000403-2/1), Arthritis Research UK (19654 and 20205), the Rosetrees Trust (A589) and NIH grant R37 HL63762 (to JH), the American Health Assistance Foundation, the Consortium for Frontotemporal Dementia Research, the Bright Focus Foundation, the Lupe Murchison Foundation and The Ted Nash Long Life Foundation.

Competing interests None declared.

Patient consent Obtained.

Provenance and peer review Not commissioned; externally peer reviewed.

Open Access This is an Open Access article distributed in accordance with the Creative Commons Attribution Non Commercial (CC BY-NC 4.0) license, which permits others to distribute, remix, adapt, build upon this work non-commercially, and license their derivative works on different terms, provided the original work is properly cited and the use is non-commercial. See: http://creativecommons.org/ licenses/by-nc/4.0/

\section{REFERENCES}

1 Ksiazek I, Burkhardt C, Lin S, et al. Synapse loss in cortex of agrin-deficient mice after genetic rescue of perinatal death. J Neurosci 2007;27:7183-95.

2 Dell'Accio F, De Bari C, Eltawil NM, et al. Identification of the molecular response of articular cartilage to injury, by microarray screening: Wnt-16 expression and signaling after injury and in osteoarthritis. Arthritis Rheum 2008;58:1410-21.

3 Choi HHY, Liu Y, Tennert C, et al. APP interacts with LRP4 and agrin to coordinate the development of the neuromuscular junction in mice. Elife 2013;2:e00220.

4 Gesemann M, Denzer AJ, Ruegg MA. Acetylcholine receptor-aggregating activity of agrin isoforms and mapping of the active site. J Cell Biol 1995;128:625-36.

5 Zhang B, Luo S, Wang Q, et al. LRP4 serves as a coreceptor of agrin. Neuron 2008;60:285-97.

6 Denzer AJ, Brandenberger R, Gesemann M, et al. Agrin binds to the nerve-muscle basal lamina via laminin. J Cell Biol 1997;137:671-83.

7 Denzer AJ, Schulthess T, Fauser $C$, et al. Electron microscopic structure of agrin and mapping of its binding site in laminin-1. EMBO J 1998;17:335-43.

8 Bezakova G, Ruegg MA. New insights into the roles of agrin. Nat Rev Mol Cell Biol 2003;4:295-308

9 Hausser $\mathrm{H}-\mathrm{JJ}$, Ruegg MA, Brenner RE, et al. Agrin is highly expressed by chondrocytes and is required for normal growth. Histochem Cell Biol 2007;127:363-74.

10 Sherwood JC, Bertrand J, Eldridge SE, et al. Cellular and molecular mechanisms of cartilage damage and repair. Drug Discov Today 2014;19:1172-7.

11 Han Y, Lefebvre V. L-Sox5 and Sox6 drive expression of the aggrecan gene in cartilage by securing binding of Sox9 to a far-upstream enhancer. Mol Cell Biol 2008;28:4999-5013.

12 Verzijl N, DeGroot J, Thorpe SR, et al. Effect of collagen turnover on the accumulation of advanced glycation end products. J Biol Chem 2000;275:39027-31.

13 Dell'Accio F, Vincent TL. Joint surface defects: clinical course and cellular response in spontaneous and experimental lesions. Eur Cell Mater 2010;20:210-17.

14 Bertrand J, Cromme C, Umlauf D, et al. Molecular mechanisms of cartilage remodelling in osteoarthritis. Int I Biochem Cell Biol 2010;42:1594-601.

15 Goldring MB, Tsuchimochi K, ljiri K. The control of chondrogenesis. J Cell Biochem 2006:97:33-44.

16 Kinds MB, Welsing PMJ, Vignon EP, et al. A systematic review of the association between radiographic and clinical osteoarthritis of hip and knee. Osteoarthritis Cartilage 2011;19:768-78.

17 Eerola I, Salminen H, Lammi P, et al. Type X collagen, a natural component of mouse articular cartilage: association with growth, aging, and osteoarthritis. Arthritis Rheum 1998:41:1287-95.

18 Wang M, Sampson ER, Jin $\mathrm{H}$, et al. MMP13 is a critical target gene during the progression of osteoarthritis. Arthritis Res Ther 2013;15:R5.

19 Bertrand J, Nitschke Y, Fuerst M, et al. Decreased levels of nucleotide pyrophosphatase phosphodiesterase 1 are associated with cartilage calcification in osteoarthritis and trigger osteoarthritic changes in mice. Ann Rheum Dis 2012:71:1249-53.

20 Glasson SS, Blanchet TJ, Morris EA. The surgical destabilization of the medial meniscus (DMM) model of osteoarthritis in the 129/SvEv mouse. Osteoarthritis Cartilage 2007;15:1061-9.

21 Nalesso G, Sherwood J, Bertrand J, et al. WNT-3A modulates articular chondrocyte phenotype by activating both canonical and noncanonical pathways. I Cell Biol 2011:193:551-64

22 Mankin HJ, Lippiello L. The glycosaminoglycans of normal and arthritic cartilage. J Clin Invest 1971:50:1712-19.

23 Dell'Accio F, De Bari C, Luyten FP. Molecular markers predictive of the capacity of expanded human articular chondrocytes to form stable cartilage in vivo. Arthritis Rheum 2001:44:1608-19.

24 De Bari C, Dell'Accio F, Luyten FP. Human periosteum-derived cells maintain phenotypic stability and chondrogenic potential throughout expansion regard less of donor age. Arthritis Rheum 2001;44:85-95.

25 Sherwood J, Bertrand J, Nalesso G, et al. A homeostatic function of CXCR2 signalling in articular cartilage. Ann Rheum Dis 2015;74:2207-15.

26 Gesemann M, Brancaccio A, Schumacher B, et al. Agrin is a high-affinity binding protein of dystroglycan in non-muscle tissue. J Biol Chem 1998;273:600-5.

27 Goldring MB, Birkhead JR, Suen L, et al. Interleukin-1 beta-modulated gene expression in immortalized human chondrocytes. J Clin Invest 1994;94:2307-16.

28 Bi W, Deng JM, Zhang Z, et al. Sox9 is required for cartilage formation. Nat Genet 1999:22:85-9.

29 De Bari C, Dell'Accio F, Luyten FP. Failure of in vitro-differentiated mesenchyma stem cells from the synovial membrane to form ectopic stable cartilage in vivo. Arthritis Rheum 2004;50:142-50.

30 Saris DBF, Vanlauwe J, Victor J, et al. Treatment of symptomatic cartilage defects of the knee: characterized chondrocyte implantation results in better clinical outcome at 36 months in a randomized trial compared to microfracture. Am I Sports Med 2009;37(Suppl 1):10S-9S.

31 Gesemann M, Cavalli V, Denzer AJ, et al. Alternative splicing of agrin alters its binding to heparin, dystroglycan, and the putative agrin receptor. Neuron 1996;16:755-67.

32 Campanelli JT, Gayer GG, Scheller RH. Alternative RNA splicing that determines agrin activity regulates binding to heparin and alpha-dystroglycan. Development 1996;122:1663-72.

33 Choi HY, Dieckmann M, Herz J, et al. Lrp4, a novel receptor for Dickkopf 1 and sclerostin, is expressed by osteoblasts and regulates bone growth and turnover in vivo. PLOS ONE 2009;4:e7930.

34 Ferns MJ, Campanelli JT, Hoch W, et al. The ability of agrin to cluster AChRs depends on alternative splicing and on cell surface proteoglycans. Neuron 1993;11:491-502.

35 Asai N, Ohkawara B, Ito M, et al. LRP4 induces extracellular matrix productions and facilitates chondrocyte differentiation. Biochem Biophys Res Commun 2014;451:302-7.

36 Johnson EB, Hammer RE, Herz J. Abnormal development of the apical ectodermal ridge and polysyndactyly in Megf7-deficient mice. Hum Mol Genet 2005;14: 3523-38

37 Kim N, Stiegler AL, Cameron TOT, et al. Lrp4 is a receptor for Agrin and forms a complex with MuSK. Cell 2008;135:334-42.

38 Yumoto N, Kim N, Burden SJ. Lrp4 is a retrograde signal for presynaptic differentiation at neuromuscular synapses. Nature 2012;489:438-42.

39 Zong Y, Zhang B, Gu S, et al. Structural basis of agrin-LRP4-MuSK signaling. Genes Dev 2012;26:247-58.

40 Ohazama A, Porntaveetus T, Ota MS, et al. Lrp4: A novel modulator of extracellular signaling in craniofacial organogenesis. Am J Med Genet A 2010; 152A:2974-83.

41 Corr M. Wnt-beta-catenin signaling in the pathogenesis of osteoarthritis. Nat Clin Pract Rheumatol 2008;4:550-6.

42 Decker RS, Koyama E, Enomoto-Iwamoto M, et al. Mouse limb skeletal growth and synovial joint development are coordinately enhanced by Kartogenin. Dev Biol 2014;395:255-67.

43 Rountree RB, Schoor M, Chen $\mathrm{H}$, et al. BMP receptor signaling is required for postnatal maintenance of articular cartilage. PLOS Biol 2004;2:e355.

44 Schett G, Zwerina J, David J-P. The role of Wnt proteins in arthritis. Nat Clin Pract Rheumatol 2008;4:473-80

45 Davidson EN, Vitters EL, Bennink MB, et al. Inducible chondrocyte-specific overexpression of BMP2 in young mice results in severe aggravation of osteophyte formation in experimental OA without altering cartilage damage. Ann Rheum Dis 2015;74:1257-64.

46 Van der Kraan PM, Blaney Davidson EN, van den Berg WB. Bone morphogenetic proteins and articular cartilage: to serve and protect or a wolf in sheep clothing's? Osteoarthritis Cartilage 2010;18:735-41.

47 Loughlin J, Dowling B, Chapman K, et al. Functional variants within the secreted frizzled-related protein 3 gene are associated with hip osteoarthritis in females. Proc Natl Acad Sci USA 2004;101:9757-62.

48 Loughlin J. Genetics of osteoarthritis. Curr Opin Rheumatol 2011;23:479-83. 